\title{
Primary debulking surgery or neoadjuvant chemotherapy followed by interval debulking surgery for patients with advanced ovarian cancer
}

\author{
Hong Zheng, Yu-Nong Gao \\ Key Laboratory of Carcinogenesis and Translational Research (Ministry of Education), Department of Gynecologic Oncology, Peking University \\ Cancer Hospital \& Institute, Beijing 100142, China \\ Corresponding to: Yu-Nong Gao. Department of Gynecologic Oncology, Peking University Cancer Hospital \& Institute, Beijing 100142, China. \\ Email: gaoyunong@vip.sina.com.
}

\begin{abstract}
Objectives: To compare the survival and perioperative morbidity between primary debulking surgery (PDS) and neoadjuvant chemotherapy followed by interval debulking surgery (NAC/IDS) in treating patients with advanced epithelial ovarian cancer (EOC).

Methods: We retrospectively reviewed 67 patients with stage IIIC or IV EOC treated at Peking University Cancer Hospital from January 2006 to June 2009. Wherein, 37 and 30 patients underwent PDS and NAC/ IDS, respectively.

Results: No difference in overall survival (OS) or progression-free survival (PFS) was observed between NAC/IDS group and PDS group (OS: 41.2 vs. 39.1 months, $\mathrm{P}=0.23$; PFS: 27.1 vs. 24.3 months, $\mathrm{P}=0.37$ ). The optimal debulking rate was $60 \%$ in the NAC/IDS group, which was significantly higher than that in the PDS group (32.4\%) ( $\mathrm{P}=0.024)$. The NAC/IDS group had significantly less intraoperative estimated blood loss and transfusion, lower nasogastric intubation rate, and earlier ambulation and recovery of intestinal function than the PDS group $(\mathrm{P}<0.05)$.

Conclusions: NAC/IDS is less invasive than PDS, and offers the advantages regarding optimal cytoreduction rate, intraoperative blood loss, and postoperative recovery, without significantly impairing the survival compared with PDS in treating patients with stage IIIC or IV EOC. Therefore, NAC/IDS may be a valuable treatment alternative for EOC patients.
\end{abstract}

Key Words: Epithelial ovarian cancer; debulking surgery; neoadjuvant chemotherapy; perioperative morbidity; survival

Submitted Jan10, 2012. Accepted for publication Aug 08, 2012.

DOI: $10.3978 /$ j.issn.1000-9604.2012.09.02

Scan to your mobile device or view this article at: http://www.thecjcr.org/article/view/1179/1516

\section{Introduction}

Ovarian cancer is the leading cause of death among gynecologic malignancies. The unfavorable prognosis of this disease is largely due to the lack of specific symptoms and early detection methods. Approximately $70 \%$ to $80 \%$ of the cases are stage III or IV when first diagnosed. The current standard treatment for epithelial ovarian cancer (EOC) consists of primary debulking surgery (PDS) followed by paclitaxel and platinum chemotherapy. Optimal cytoreductive surgery with residual disease less than 1 or $2 \mathrm{~cm}$ is one of the most important prognostic factors to predict survival in patients with advanced EOC (1). However, stage III or IV disease, which means the tumor has disseminated to the whole abdomen, or distant organ parenchyma, makes optimal cytoreductive surgery aggressive and occasionally hard to accomplish. In these cases, radical surgical procedures, such as splenectomy, bowel resection and partial hepatectomy, are often required. The optimal cytoreduction rate is only around $50 \%$ and could be less than $25 \%$ despite maximal efforts (2). Most patients 
diagnosed with advanced EOC are elderly, with multiple comorbidities. These patients usually have poor nutritional status due to large volume of ascites. Therefore, aggressive surgery is significantly limited in these patients.

Neoadjuvant chemotherapy (NAC) is a chemotherapy method for primary solid tumors before surgical ablation. Ovarian cancer is a chemosensitive tumor with a response rate of $70 \%$ to $80 \%$ (3), thus, NAC can usually result in adequate tumor shrinkage and increase the possibility of optimal tumor debulking. Therefore, NAC followed by interval debulking surgery (NAC/IDS) has been considered as an alternative to conventional PDS in treating advanced EOC.

In the last 20 years, over 30 studies regarding NAC in advanced ovarian cancer were conducted, most of which are retrospective in nature. The effect of NAC/IDS on survival and perioperative morbidity varies in these studies (4-6). According to these studies, patients treated with NAC/IDS had comparable survival with those who underwent PDS (5,7-9).

In the present study, we compared the survival and perioperative morbidity between patients with stage IIIC or IV EOC who were treated with PDS and NAC/IDS.

\section{Materials and methods}

\section{Patients and treatment}

We retrospectively reviewed 67 patients diagnosed with stage IIIC or IV EOC who were treated at Peking University Cancer Hospital from January 2006 to June 2009. The study was approved by the Institutional Review Board. Thirtyseven patients received PDS and 30 underwent NAC/IDS. The diagnosis for the NAC-treated patients was based on laparoscopic biopsy (53\%), ultrasound-guided biopsy (30\%), or cytology (17\%).

NAC was given when optimal cytoreduction was considered infeasible by computerized tomography (CT) scan or when the patients were in poor conditions [Karnofsky Performance Scale (KPS) scores under 60]. NAC-treated patients usually presented a great amount of ascites, extensive bowel mesenteric involvement, or diffused peritoneal thickening, as indicated by CT scans.

In the NAC/IDS group, most patients were given two courses of chemotherapy (83.3\%) before the debulking surgery, whereas the others were given one course. Twentyfive patients received paclitaxel/carboplatin (CP), 4 received cyclophosphamide/doxorubicin/carboplatin (CAC), and 1 received cyclophosphamide/cisplatin as NAC regimens.
Paclitaxel was given at $175 \mathrm{mg} / \mathrm{m}^{2}$ in association with carboplatin at the area under the curve of 5 . Doxorubicin was given at $50 \mathrm{mg} / \mathrm{m}^{2}$, cyclophosphamide at $600 \mathrm{mg} / \mathrm{m}^{2}$, and cisplatin at $75 \mathrm{mg} / \mathrm{m}^{2}$. Courses were repeated every 3 weeks.

All patients underwent debulking surgery after NAC, and were given the same regimen as that of adjuvant chemotherapy for another 4-6 cycles postoperatively.

After standard debulking surgery, patients in the PDS group received 6-8 cycles of carboplatin and paclitaxel. However, the second-line chemotherapy was administered if the disease progressed. All chemotherapy regimens were given intravenously.

Optimal cytoreduction was considered as no residual tumor mass greater than $1 \mathrm{~cm}$. The patients were followed up every 3-6 months after treatment. The mean follow-up time was 31.6 months (range, 14-55 months); the last followup date was August 31, 2010. Four patients were lost during the follow-up period, 1 in NAC/IDS arm and 3 in PDS arm.

\section{Statistical analysis}

Overall survival (OS) and progression-free survival (PFS) were analyzed using the Kaplan-Meier method. The logrank test was used to investigate the difference in survival between the study groups. Cox proportional hazards analysis was used to evaluate the prognostic factors that affect survival. Parameters assessing perioperative morbidities were compared using the Student's $t$-test or cross tabulations. The statistical analyses were performed with SPSS software version 16.0 (SPSS Inc., Chicago, IL, USA). $\mathrm{P}<0.05$ was considered statistically significant.

\section{Results}

\section{Patients' characteristics}

Patient profiles are shown in Table 1. The age, tumor grade and histological characteristics were evenly distributed between two groups. The number of patients with stage IV disease were greater in the NAC/IDS group $(\mathrm{P}=0.024)$.

\section{Response to NAC}

The amount of ascites in 25 patients and the volume of pleural effusions in 3 patients were well controlled by NAC. All patients exhibited symptom relief to some extent, and their KPS scores significantly improved after NAC (90 vs. 60, $\mathrm{P}=0.00$ ). Using the CA125 criteria (Gynecologic Cancer 


\begin{tabular}{|c|c|c|c|}
\hline Variables & $\operatorname{NAC}(n=30)$ & $\operatorname{PDS}(n=37)$ & $\mathrm{P}$ \\
\hline Age (mean, year) & 55.8 & 54.5 & 0.58 \\
\hline \multicolumn{4}{|l|}{ FIGO stage } \\
\hline IIIC & 20 (66.7\%) & $33(89.2 \%)$ & \multirow{2}{*}{0.024} \\
\hline IV & 10 (33.3) & $4(10.8 \%)$ & \\
\hline \multicolumn{4}{|l|}{ Histology } \\
\hline Serous & 22 (73.3\%) & $30(81.1 \%)$ & \multirow{2}{*}{0.45} \\
\hline Non-serous & $8(26.7 \%)$ & 7 (18.9\%) & \\
\hline \multicolumn{4}{|c|}{ Histological grade } \\
\hline 1 & 0 & $1(2.7 \%)$ & \multirow{3}{*}{0.33} \\
\hline 2 & $4(13.3 \%)$ & 9 (24.3\%) & \\
\hline 3 & $26(86.7 \%)$ & 27 (73.0\%) & \\
\hline
\end{tabular}

FIGO, International Federation of Gynecology and Obstetrics

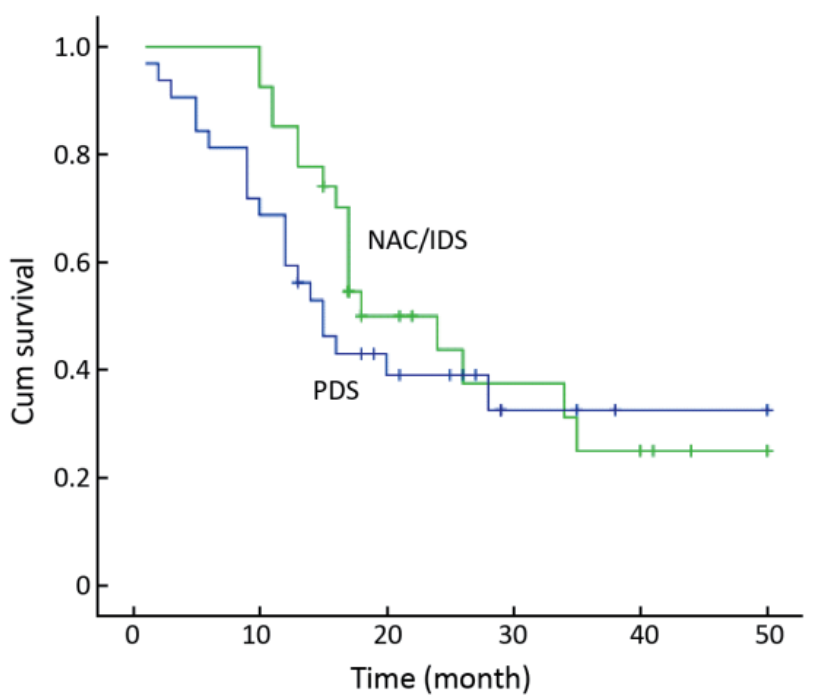

Figure 2 Kaplan-Meier survival curve showing PFS between NAC/IDS and PDS groups

Intergroup criteria) (10), 12 patients achieved complete response (CR), 16 patients had partial response (PR), and 2 patients had stable disease (SD).

Significantly higher optimal cytoreduction rate was achieved in the NAC/IDS group than that in the PDS group (60\% vs. $32.4 \%, \mathrm{P}=0.024)$.

\section{Comparison of survival between NAC/IDS and PDS groups}

The mean OS was 41.2 [95\% confidence interval (CI):

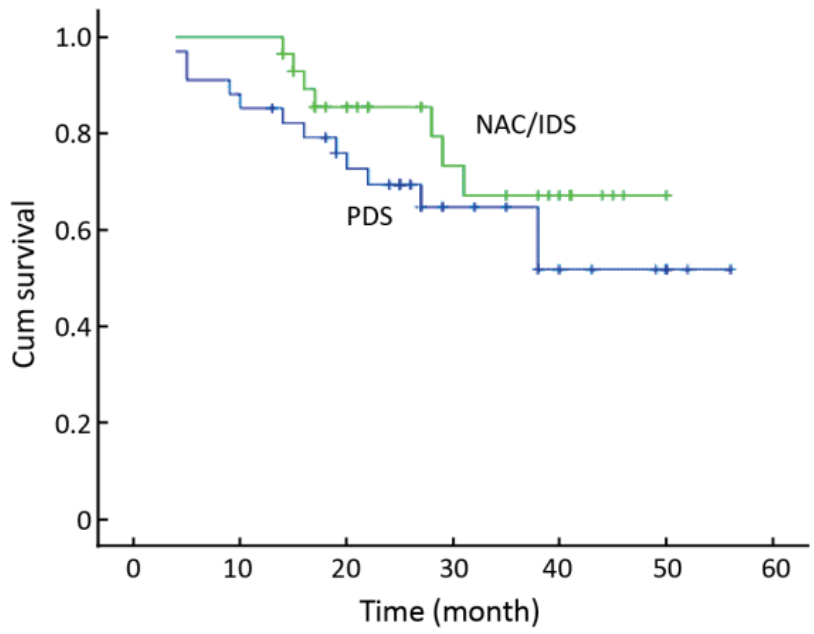

Figure 1 Kaplan-Meier survival curve showing OS between NAC/ IDS and PDS groups

35.75-46.71] months in the NAC/IDS group and 39.1 (95\% CI: 31.96-46.19) months in the PDS group. Seven patients in the NAC/IDS group and 13 in the PDS group died of the disease during the follow-up period. No statistically significant difference was found in OS between the NAC/ IDS and PDS groups $(\mathrm{P}=0.23)$ (Figure 1). The mean PFS was 27.1 (95\% CI: 20.9-33.3) months in the NAC/IDS group and 24.3 (95\% CI: 17.4-31.1) months in the PDS group, which was not significantly different between the two study groups $(\mathrm{P}=0.37)$ (Figure 2).

The patients with stage IV disease were not evenly distributed between the two study groups, thus, we performed multivariate Cox regression analyses which showed that NAC/IDS did not affect OS $(\mathrm{P}=0.06)$, while younger age $(\mathrm{P}=0.036)$, earlier stage $(\mathrm{P}=0.002)$ and residual tumor mass less than $1 \mathrm{~cm}(\mathrm{P}=0.004)$ were independent prognostic factors that predict improved survival.

In the NAC/IDS group, the mean OS in patients with normalized CA125 after NAC was similar to those with abnormal CA125 after NAC (47.0 vs. 36.4 months, $\mathrm{P}=0.20$ ). The PFS was also similar between patients with normalized and abnormal CA125 after NAC (33.1 vs. 22.8 months, $\mathrm{P}=0.18)$.

\section{Comparison of perioperative parameters between NAC/ IDS and PDS groups}

The perioperative parameters evaluating the surgical proceedings and recovery from the surgery are listed 
Table 2 Comparison of perioperative parameters between NAC/IDS and PDS groups

\begin{tabular}{lccc} 
Parameters & NAC & PDS & P \\
\hline Estimated blood loss $(\mathrm{mL})$ & 415.0 & 729.7 & 0.014 \\
Transfusion $(\mathrm{U})$ & 1.87 & 2.97 & 0.033 \\
Operation time (min) & 168.7 & 181.7 & 0.486 \\
Nasogastric intubation (\%) & 13.3 & 43.2 & 0.008 \\
Total parenteral nutrition (d) & 0.47 & 1.54 & 0.016 \\
Ambulation post-operation (d) & 1.57 & 2.65 & 0.001 \\
Abnormal temperature (d) & 2.97 & 3.70 & 0.250 \\
Recovery of intestinal function (d) & 3.0 & 3.5 & 0.031 \\
\hline
\end{tabular}

in Table 2. The patients treated with NAC/IDS showed less estimated blood loss and a smaller amount of blood transfusion than those treated with conventional PDS. Fewer patients needed nasogastric intubation and total parenteral nutrition in the NAC/IDS group than that in the PDS group. The patients who underwent NAC/IDS had early ambulation and improved recovery of intestinal function. Postoperative complications including cardiac accident, bowel or urinary injury, wound separation, ileus, and venous thrombosis were comparable between the NAC/ IDS and PDS groups (16.7\% vs. 24.3\%, P>0.05).

\section{Discussion}

PDS followed by adjuvant chemotherapy is still the standard of care for advanced stage EOC patients. The amount of residual tumor after PDS is an important prognostic factor for survival in several retrospective and prospective studies $(1,11)$. Thus, great efforts including radical surgical procedures have been made to achieve optimal cytoreduction. Majority of patients with advanced EOC are elderly and in poor performance status due to large tumor volume and ascites, thus, extensive debulking surgery is sometimes limited. NAC/IDS is an alternative treatment for these patients. No consensus has been made on the criteria of selecting patients who can be treated with NAC/IDS. In most studies, patients in poor performance status or considered to have non-cytoreducible disease received NAC. CT scan is the most widely used tool to evaluate the extent of the disease. However, CT scan has insufficient accuracy to predict the cytoreductive outcome. Preoperative assessment and defining criteria for selecting patients who may benefit from NAC/IDS are warranted as PDS is still the standard of care if optimal cytoreduction can be achieved

It was demonstrated in many studies that NAC/ IDS effectively increases the feasibility of optimal cytoreductive surgery in advanced EOC (12-15). However, the most important question on whether NAC/IDS is superior to PDS in survival is still under investigation. The majority of the studies showed that NAC/IDS had no impact on OS compared with PDS, including a meta-analysis of 21 studies and a recently published randomized trial $(5,8,9,13,14,16)$. NAC/IDS was found to improve survival $(4,12,17)$. However, a metaanalysis pointed out that NAC/IDS survival outcomes were inferior compared with conventional primary surgery (18). Although the NAC/IDS group had more patients with stage IV disease, which was an independent negative prognostic factor in our multivariate analysis, the patients treated with NAC/IDS did not have impaired PFS and OS compared with those treated with conventional PDS. This result is consistent with most studies. The survival result is partly explained by the higher percentage of patients with optimal cytoreduction in the NAC/IDS group.

Although no survival advantage is offered by NAC/IDS, this treatment method provides favorable perioperative morbidity. In our study, the patients treated with NAC/ IDS had less estimated blood loss during the operation and quicker recovery in terms of intestinal function and ambulation. These results are consistent with previous studies. Chan et al. reported that NAC improved the patients' overall quality of life and functional status (19). Other studies also reported that patients who underwent NAC/IDS had lower estimated blood loss and shorter OR time and hospital stay $(12,14,20)$. Only a few studies found no difference in perioperative morbidity between the NAC/ IDS and PDS groups (4).

Only a few patients repeated the CT scan after NAC due to financial reasons. The response of NAC in most patients was evaluated by serum CA125 level or physical examination. CA125 is a good surrogate marker for tumor response, and the patients with normalized CA125 after neoadjuvant chemotherapy had a better survival (21). In our small-sized study, normalized CA125 after NAC was not a prognostic factor of OS or PFS. Thus, giving NAC until CA125 is normalized is not helpful in improving survival. A study by Le et al. also revealed that CA125 normalization after neoadjuvant chemotherapy is not an independent predictor of either PFS or OS (22). In their meta-analysis, Bristow and Chi found that increasing the number of chemotherapy cycles prior to the debulking surgery 
had a negative survival effect. Thus, definitive operative intervention should be undertaken early in the treatment program as possible (18). However, a more recent metaanalysis did not show that increasing the number of NAC cycles adversely affected OS (8). The best time to perform cytoreductive surgery after NAC is still unclear and needs more investigation.

In conclusion, NAC/IDS provides equal survival compared with conventional PDS. NAC/IDS is also a relatively safe approach to achieve optimal cytoreduction in patients with non-optimally cytoreducible disease or in poor performance status. NAC/IDS can be an alternative to PDS in treating stage IIIC or IV EOC. Investigations aimed at appropriate selection of patients to be treated with NAC and identification of the best time to perform IDS will provide greater benefits for patients with advanced EOC.

\section{Acknowledgements}

Disclosure: The authors declare no conflict of interest.

\section{References}

1. Bristow RE, Tomacruz RS, Armstrong DK, et al. Survival effect of maximal cytoreductive surgery for advanced ovarian carcinoma during the platinum era: a metaanalysis. J Clin Oncol 2002;20:1248-59.

2. Gallo A, Frigerio L. Neoadjuvant chemotherapy and surgical considerations in ovarian cancer. Curr Opin Obstet Gynecol 2003;15:25-31.

3. McGuire WP, Hoskins WJ, Brady MF, et al. Cyclophosphamide and cisplatin compared with paclitaxel and cisplatin in patients with stage III and stage IV ovarian cancer. N Engl J Med 1996;334:1-6.

4. Kuhn W, Rutke S, Späthe K, et al. Neoadjuvant chemotherapy followed by tumor debulking prolongs survival for patients with poor prognosis in International Federation of Gynecology and Obstetrics Stage IIIC ovarian carcinoma. Cancer 2001;92:2585-91.

5. Morice P, Dubernard G, Rey A, et al. Results of interval debulking surgery compared with primary debulking surgery in advanced stage ovarian cancer. J Am Coll Surg 2003;197:955-63.

6. Vergote I, De Wever I, Tjalma W, et al. Neoadjuvant chemotherapy or primary debulking surgery in advanced ovarian carcinoma: a retrospective analysis of 285 patients. Gynecol Oncol 1998;71:431-6.

7. Loizzi V, Cormio G, Resta L, et al. Neoadjuvant chemotherapy in advanced ovarian cancer: a case-control study. Int J Gynecol Cancer 2005;15:217-23.

8. Kang S, Nam BH. Does neoadjuvant chemotherapy increase optimal cytoreduction rate in advanced ovarian cancer? Meta-analysis of 21 studies. Ann Surg Oncol 2009;16:2315-20.

9. Vergote I, Tropé CG, Amant F, et al. Neoadjuvant chemotherapy or primary surgery in stage IIIC or IV ovarian cancer. N Engl J Med 2010;363:943-53.

10. Vergote I, Rustin GJ, Eisenhauer EA, et al. Re: new guidelines to evaluate the response to treatment in solid tumors [ovarian cancer]. Gynecologic Cancer Intergroup. J Natl Cancer Inst 2000;92:1534-5.

11. Elattar A, Bryant A, Winter-Roach BA, et al. Optimal primary surgical treatment for advanced epithelial ovarian cancer. Cochrane Database Syst Rev 2011;8:CD007565.

12. Hou JY, Kelly MG, Yu H, et al. Neoadjuvant chemotherapy lessens surgical morbidity in advanced ovarian cancer and leads to improved survival in stage IV disease. Gynecol Oncol 2007;105:211-7.

13. Everett EN, French AE, Stone RL, et al. Initial chemotherapy followed by surgical cytoreduction for the treatment of stage III/IV epithelial ovarian cancer. Am J Obstet Gynecol 2006;195:568-74; discussion 574-6.

14. Lee SJ, Kim BG, Lee JW, et al. Preliminary results of neoadjuvant chemotherapy with paclitaxel and cisplatin in patients with advanced epithelial ovarian cancer who are inadequate for optimum primary surgery. J Obstet Gynaecol Res 2006;32:99-106.

15. Vrscaj MU, Rakar S. Neoadjuvant chemotherapy for advanced epithelial ovarian carcinoma: a retrospective case-control study. Eur J Gynaecol Oncol 2002;23:405-10.

16. Rauh-Hain JA, Rodriguez N, Growdon WB, et al. Primary debulking surgery versus neoadjuvant chemotherapy in stage IV ovarian cancer. Ann Surg Oncol 2012;19:959-65.

17. Rafii A, Deval B, Geay JF, et al. Treatment of FIGO stage IV ovarian carcinoma: results of primary surgery or interval surgery after neoadjuvant chemotherapy: a retrospective study. Int J Gynecol Cancer 2007;17:777-83.

18. Bristow RE, Chi DS. Platinum-based neoadjuvant chemotherapy and interval surgical cytoreduction for advanced ovarian cancer: a meta-analysis. Gynecol Oncol 2006;103:1070-6.

19. Chan YM, Ng TY, Ngan HY, et al. Quality of life in women treated with neoadjuvant chemotherapy for advanced ovarian cancer: a prospective longitudinal study. Gynecol Oncol 2003;88:9-16.

20. Glasgow MA, Yu H, Rutherford TJ, et al. Neoadjuvant chemotherapy (NACT) is an effective way of managing elderly women with advanced stage ovarian cancer (FIGO Stage IIIC and IV). J Surg Oncol 2012. [Epub ahead of print]. 
21. Mazzeo F, Berlière $M$, Kerger J, et al. Neoadjuvant chemotherapy followed by surgery and adjuvant chemotherapy in patients with primarily unresectable, advanced-stage ovarian cancer. Gynecol Oncol 2003;90:163-9.

Cite this article as: Zheng H, Gao YN. Primary debulking surgery or neoadjuvant chemotherapy followed by interval debulking surgery for patients with advanced ovarian cancer. Chin J Cancer Res 2012;24(4):304-309. DOI: 10.3978/ j.issn.1000-9604.2012.09.02
22. Le T, Faught W, Hopkins L, et al. Importance of CA125 normalization during neoadjuvant chemotherapy followed by planned delayed surgical debulking in patients with epithelial ovarian cancer. J Obstet Gynaecol Can 2008;30:665-70. 